\title{
Lactobacillus casei Zhang Ameliorates Blood-Milk Barrier Disruption and Suppresses the Inflammatory Response during Escherichia coli-induced Mastitis
}

\author{
Yuhui Zheng ${ }^{1}$, gang liu $^{1}$, wei wang ${ }^{1}$, yajing wang ${ }^{1}$, zhijun cao ${ }^{1}$, hongjian yang $^{1}$, and shengli \\ $\mathrm{li}^{1}$ \\ ${ }^{1}$ China Agricultural University College of Animal Science and Technology
}

August 28, 2020

\begin{abstract}
Background and Purpose: Escherichia coli is a common mastitis-causing pathogens and is destructive to the blood-milk barrier. Oral administration of Lactobacillus casei Zhang (LCZ) could alleviate mice mastitis. However, its prophylactic effect and mechanism through intramammary injection on E. coli-induced mastitis is unclear now. Here, we investigate this using E. coliinduced mastitis model. Experimental Approach: Prophylactic effects and mechanism of intramammary injection of LCZ on blood-milk barrier and inflammation were studied in both bovine mammary epithelial cells (BMECs) and pregnant mice. Key Results: In vitro tests revealed that LCZ significantly inhibited the adhesion of E. coli to monolayer cells, reduce the damage of cell desmosomes, up-regulated the expression of tight junction proteins (claudin-1, claudin-4, occludin, and ZO-1), and downregulated the expression of inflammatory cytokines (TNF- $\alpha$, IL-1 $\beta$, and IL-6) thereby enhancing the trans-epithelial electric resistance of monolayer BMECs and effectively protecting cells from damage caused by E. coli. In vivo experiments suggested that LCZ significantly promoted the expression of tight junction proteins (claudin-3, occludin, and ZO-1) but significantly inhibited the expression of inflammatory cytokines (TNF- $\alpha$, IL-1 $\beta$, and IL-6) in mouse mammary tissue, thereby decreased disruption in mammary tissues, infiltration of inflammatory cells in E. coli-induced mastitis. Conclusions and Implications: In our study, LCZ ameliorates blood-milk barrier disruption and suppresses the inflammatory response during E. coli-induced mastitis, indicating LCZ may serve as a effective prophylactic agent to preserve the blood-milk barrier function during mastitis.
\end{abstract}

\section{INTRODUCTION}

Bovine mastitis is a common disease on dairy farms and leads to enormous economic losses worldwide (Angelopoulou et al., 2018; Sathiyabarathi et al., 2016). Escherichia coli is one of the most common mastitiscausing pathogens and often leads to acute mastitis with a severe inflammatory response that damages the blood-milk barrier. The abuse of antimicrobials in food animals has attracted public attention as concerns relating to antimicrobial residues and anti-microbial resistance in bacteria have increased because of the increased use of antimicrobials (Muziasari et al., 2016; Lhermie et al., 2016). Therefore, there is an urgent need to find new potential non-antimicrobials for mastitis prevention.

Lactobacillus is a probiotic that plays an important role in the immunity and health of animals. Their probiotic properties play an important role in regulating gut microbiota (Igor et al., 2019), relieving intestinal diseases (Sanders et al., 2019), and enhancing body immunity (Garcia-Castillo et al., 2019) in addition to other functions (Li et al., 2017; da Costa et al., 2019). Lactobacillus is known to protect hosts during intramammary infection in both humans and cows by oral or intramammary administration (Fernández et al., 2016; Arroyo et al., 2010; Jiménez et al., 2008), but the underlying mechanism of this protective effect remains unclear. Intramammary injection of drugs is the most common and effective way for treating bovine mastitis in practice, as intramammary injection of some Lactobacillus strains can effectively alleviate 
clinical symptoms of bovine mastitis (Ignacio et al., 2015; Pellegrino et al., 2017; Kitching et al., 2019). Oral administration of Lactobacillus casei Zhang ( $\mathbf{C C Z}$ ) isolated from naturally fermented mare's milk is effective for alleviating the clinical symptoms of mastitis in mice (Ma et al., 2018). However, whether intramammary injection of LCZ has a prophylactic effect on mastitis, as well as its underlying mechanism, remains unclear. Here, we studied the prophylactic effect of LCZ by intramammary injection on $E$. coli -induced mastitis.

\section{METHODS}

Bacterial strains and growth conditions

Lactobacillus casei Zhang (provided by Inner Mongolia Agricultural University Key Laboratory of Dairy Biotechnology and Engineering, Inner Mongolia, China) was grown in De Man, Rogosa, and Sharpe (MRS) broth (Solarbio Life Science, Beijing, China) at 37 under microaerophilic conditions. The isolate of E. coli ATCC 25922 (China General Microbiological Culture Collection Center, Beijing, China) was incubated in Tryptone Soya Broth (TSB, Solarbio Life Science, Beijing, China) at 37. For all treatments, E. coli and LCZ were plated on Tryptose Soya Agar (TSA, Solarbio Life Science, Beijing, China) and MRS agar (Solarbio Life Science, Beijing, China), respectively, after serial dilution and quantified by determination of colony-forming units (CFU).

\section{Cell culture}

Bovine mammary epithelial cells (BMECs; provided by Yangzhou University, College of Animal Science and Technology Feed Engineering Technology Research Center, China) were cultured in DMEM-F12 medium (ThermoFisher Scientific, Waltham, MA, USA) containing 10\% FBS (ThermoFisher Scientific, Waltham, MA, USA), $1 \%$ penicillin and streptomycin (Beyotime Biotechnology, Shanghai, China), $15 \mathrm{ng} / \mathrm{mL}$ EGF (PeproTech, Cranbury, NJ, USA), $1 \%$ non-essential amino acids (ThermoFisher Scientific, Waltham, MA, USA), and 1\% insulin-transferrin-selenium (ThermoFisher Scientific, Waltham, MA, USA) at 37 in an atmosphere of $5 \% \mathrm{CO}_{2}$ and $95 \%$ air at $95 \%$ relative humidity. Briefly, BMECs $\left(5 \times 10^{5}\right.$ ) were seeded onto six-well cell-culture plates in the aforementioned medium for $48 \mathrm{~h}$ and were then cultured in DMEM-F12 medium containing 5\% FBS, $1 \%$ penicillin and streptomycin, and 1\% ITS for $72 \mathrm{~h}$ to achieve a polarized and differentiated state. Before treatments, the medium was changed into DMEM-F12 alone, and bothE. coli and LCZ were diluted by DMEM-F12. Cells were randomly treated under one of four conditions as follows: (i) Control group (DMEM alone); (ii) $1 \times 10^{3}$ CFU E. coli treatment for $3 \mathrm{~h}$ (E. coli group); (iii) $1 \times 10^{5}$ CFU LCZ pretreatment for $5 \mathrm{~h}$, followed by $1 \times 10^{3} \mathrm{CFU}$ E. coli treatment for $3 \mathrm{~h}$ (LCZ + E. coli group); and (iv) $1 \times 10^{5} \mathrm{CFU}$ LCZ treatment for $5 \mathrm{~h}$ (LCZ group). At $3 \mathrm{~h}$ after pretreatment, the cells were washed three times with PBS (ThermoFisher Scientific, Waltham, MA, USA) and exposed to E. coli .

Cell death assay

Cell death was measured using the lactate dehydrogenase (LDH) method through a Cytotox 96 cytotoxicity assay (Solarbio Life Science, Beijing, China) per the manufacturer's manual.

Adhesion assay

The BMECs were washed with PBS three times, and one milliliter triton-X-100 $(0.5 \% \mathrm{~V} / \mathrm{V}$, Solarbio Life Science, Beijing, China) was added in each well after incubation with LCZ or E. coli . The cells were scraped off, and the lysate was transferred to a $1.5-\mathrm{mL}$ centrifuge tube after $10 \mathrm{~min}$ of stabilization at room temperature.E. coli and LCZ were plated on TSA and MRS agar, respectively, after serial dilution and were quantified by determination of CFU.

Measurement of the trans-epithelial electric resistance

To measure the trans-epithelial electric resistance (TEER) of monolayer BMECs, BMECs were seeded to confluence on transwell inserts (membrane area $0.33 \mathrm{~cm}^{2}$, pore size $0.4 \mu \mathrm{m}$, Corning, NY, USA) and a polarized and differentiated state was achieved within 5 days after seeding. TEER was measured immediately after different treatments with a Millicell Electrical Resistance System-2 (Millipore, MA, USA). Measurements of TEER were made three times per well and expressed as $\Omega[?] \mathrm{cm}^{2}$ after subtracting the filter resistance value. 
The results of TEER were expressed as change of TEER (TEER of monolayer cells after treatments - TEER of monolayer cells before treatments).

Animals

Pregnant CD-1 mice (8-10 days in lactation; $50 \pm 5$ g; purchased from SPF Biotechnology Co., Ltd, Beijing, China) were housed at room temperature with a $12 \mathrm{~h}$ light/12 h dark cycle and had free access to food and water in China Agricultural University Laboratory Center (Beijing, China). All animal care and experimental protocols in the study were conducted in accordance with the animal ethics committee of the National Institutes of Health Guide for the Care and Use of Laboratory Animals (GB14925-2010, China). Twenty mice were randomly treated under one of four conditions as follows: (i) Control group (PBS alone); (ii) $1 \times$ $10^{3} \mathrm{CFU}$ E. coli treatment for $12 \mathrm{~h}$ (E. coli group); (iii) $1 \times 10^{5} \mathrm{CFU}$ LCZ pre-injection for $24 \mathrm{~h}$, followed by $1 \times 10^{3} \mathrm{CFUE}$. coli treatment for $12 \mathrm{~h}$ (LCZ + E. coli group); and (iv) $1 \times 10^{5}$ CFU LCZ treatment for $24 \mathrm{~h}$ (LCZ group). Both LCZ and E. coli were diluted by PBS. Mice were anesthetized with Zoletil 50 (Virbac, Barneveld, France), and all treatments were conducted through intramammary injection via the teat canal of the fourth teat. E. coli was injected $24 \mathrm{~h}$ after LCZ pretreatment. After treatments, the mice were sacrificed by cervical dislocation, and the mammary tissues were collected.

Histopathology

The general condition of each mammary tissue of mice was assessed by clinical scoring. The clinical score ranked from 1 to 5 , with higher scores corresponding to greater degrees of tissue damage. Specifically, 1 indicated no damage, 2 indicated slight redness, 3 indicated redness and slight bleeding, 4 indicated redness and bleeding, and 5 indicated redness and obvious bleeding.

The mammary tissues were fixed in $4 \%$ cell tissue fixative (Solarbio Life Science, Beijing, China) immediately after the mice were sacrificed. Tissues were embedded in paraffin and sliced into 5 - $\mu$ m slices. To evaluate histological changes, tissues were stained with hematoxylin-eosin and observed under a microscope. The semi-quantitative scoring was performed using a histological score based on the following criteria. The histological score ranged from 1 to 5, and higher scores corresponded to greater degrees of tissue damage. Specifically, 1 indicated the absence of histological features (e.g., necrosis, neutrophils, and lymphocytes), 2 indicated minimal histological features (i.e., individual neutrophils), 3 indicated mild histological features (i.e., a small amount of neutrophils), 4 indicated moderate histological characteristics (i.e., many neutrophils and slight damage to glandular structure), and 5 indicated severe histological characteristics (i.e., a large number of neutrophils and severe damage to glandular structure). Both clinical and histological scoring was made by experienced veterinary pathologists who were blinded to treatments.

Immunofluorescence

Two mammary tissue blocks $\left(0.3 \mathrm{~cm}^{3}\right)$ were immediately sampled after the mice were sacrificed and then stored in liquid nitrogen for tissue immunofluorescence. Briefly, 5 - $\mu \mathrm{m}$ cryosections of mammary glands were fixed with $4 \%$ paraformaldehyde in PBS for $40 \mathrm{~min}$ at room temperature, followed by incubation with PBS containing 5\% BSA (ThermoFisher Scientific, Waltham, MA, USA) to block nonspecific interactions and then treatment with primary antibodies diluted by BSA overnight at 4 . After washing with PBS, the sections were exposed to secondary antibodies and diluted by $5 \%$ BSA for $1 \mathrm{~h}$ at room temperature. Controls were treated in the same manner, except that primary antibodies were not applied. After washing with PBS and staining with DAPI for 5 min, images of stained sections were obtained using a fluorescence microscope.

Transmission electron microscope analysis

Two mammary gland tissue blocks $\left(10 \mathrm{~mm}^{3}\right)$ collected from each mouse were immediately stored in centrifuge tubes and filled with electron microscope fixative for transmission electron microscopy (TEM) (Gang et al., 2019). Briefly, cell samples were harvested and filled with an electron microscope fixative for TEM. Both tissue and cell samples were dehydrated with ethanol (30, 50, 70, 80, 90, and 100\% ethanol) with 15 min per step at room temperature. Cells were then embedded in epoxy resin acetone mixtures $(2: 1)$ for $2 \mathrm{~h}$ and in pure resin at $37{ }^{\circ} \mathrm{C}$ overnight. Next, ultra-thin sections were cut using an ultramicrotome (Leica 
EM, Germany), stained with $1 \%$ uranyl acetate and lead citrate, and examined with TEM (Hitachi H-7650, Japan).

Western blot analysis

Both BMECs and mammary tissue samples were obtained after treatment, and whole protein was extracted using a Total Protein Extraction Kit (Beyotime Biotechnology, Shanghai, China). The protein concentrations were determined using a BCA Protein Assay Kit (Beyotime Biotechnology, Shanghai, China). Each sample (40 $\mu \mathrm{g}$ of total protein) was separated by $12 \%$ SDS polyacrylamide gels and transferred onto PVDF membranes (Bio-Rad, CA, USA). The membranes were blocked with $5 \%$ BSA in $0.05 \%$ Tris-buffered saline with Tween (TBST, Nobleryder, Beijing, China) overnight at 4 . The membranes were then incubated with primary antibodies (ZO-1, claudin-1, claudin-4, and occludin for BMECs, 1:500 dilution, Bioss Antibodies, Beijing, China; ZO-1, claudin-3, and occludin for mice mammary tissue, 1:2000 dilution, Abcam, Cambridge, UK) at room temperature for $3 \mathrm{~h}$. After washing with TBST, incubation with secondary antibodies (1:1000 dilution, CoWin Biosciences, Beijing, China) was performed for $1 \mathrm{~h}$ at room temperature. Protein bands were visualized using a Beyo Enhanced Chemiluminescence Reagent Kit (Solarbio Life Science, Beijing, China). The volume of the protein bands was measured by MultiImager (Bio-Rad Gel Doc XR, CA, USA) and ImageJ software (National Institutes of Health, Bethesda, MD, USA).

\section{ELISA assay}

The expression of TNF- $\alpha$, IL-6, and IL- $1 \beta$ in BMECs and mammary tissues of mice was quantified using bovine ELISA kits (DG Biotech, Beijing, China) and mouse ELISA kits (ThermoFisher Scientific, Waltham, MA, USA), respectively.

Statistical analysis

All data analyses were performed in SAS 9.2 (SAS Institute Inc., NC, USA), and graphs were produced with Origin 8.0 (Origin Lab, MA, USA). Data were presented as mean \pm SEM. Analysis of variance was performed using the ANOVA procedure in SAS 9.2. If the differences between treatments were significant, multiple comparisons were performed using the Tukey method. Statistical significance was considered $\mathrm{P}<$ 0.05 .

\section{Material}

MRS broth, TSB, TSA, MRS agar, 4\% cell tissue fixative and Beyo Enhanced Chemiluminescence Reagent Kit were all purchased from Solarbio Life Science (Beijing, China). DMEM-F12 medium, FBS, 1\% nonessential amino acids, $1 \%$ insulin-transferrin-selenium, PBS, BSA, and mouse TNF- $\alpha$, IL-6, and IL-1 $\beta$ ELISA kits were all purchased from ThermoFisher Scientific (Waltham, MA, USA). 1\% penicillin and streptomycin, Total Protein Extraction Kit, and BCA Protein Assay Kit were purchased from Beyotime Biotechnology (Shanghai, China). Bovine TNF- $\alpha$, IL-6, and IL-1 $\beta$ ELISA kits was purchased from DG Biotech (Beijing, China), $15 \mathrm{ng} / \mathrm{mL}$ EGF was purchased from PeproTech (Cranbury, NJ, USA), and TBST was purchased from Nobleryder (Beijing, China). Primary antibodies for western blot (ZO-1, claudin-1, claudin-4, and occludin for BMECs) were purchased from Bioss Antibodies (Beijing, China), and ZO-1, claudin-3, and occludin for mice mammary tissue were purchased from Abcam, (Cambridge, UK). All secondary antibodies for western blot were purchased from CoWin Biosciences (Beijing, China).

\section{RESULTS}

Effect of LCZ on cell growth and the blood-milk barrier

E. coli caused a significantly higher LDH release compared with other groups. No significant difference in $\mathrm{LDH}$ concentration was observed in the $\mathrm{LCZ}+E$. coli and LCZ groups compared with the Control group (Figure 1A).

The change in TEER in BMECs treated with E. coli was negative and significantly lower than that of the other groups; specifically, the change in TEER was negative in the LCZ + E. coli group but significantly 
less negative compared with the change in TEER observed in the E. coli group. In addition, the change of TEER in the LCZ group was significantly higher than that of the Control group (Figure 1B).

The desmosome structure in the E. coli treatment group was severely damaged and blurred, as illustrated by TEM. Meanwhile, the degree of damage to the desmosome structure alleviated was noticeably greater in the $\mathrm{LCZ}+E$. coli group compared with the E. coli group. Moreover, the desmosome structure in the LCZ group was clearly visible under TEM, and no clear difference was detected compared with the Control group (Figure 1C).

\section{Effect of $L C Z$ on the adhesion rate of $\mathbf{E}$. coli to $\boldsymbol{B M E C s}$}

The adhesion rate of $E$. coli to monolayer BMECs in the LCZ $+E$. coli group was significantly lower than that of the E. coli group. In addition, the adhesion rate of LCZ to monolayer BMECs was significantly lower than that of E. coli (Figure 2).

Effect of LCZ on the expression of the tight junction proteins of BMECs

The expression of tight junction proteins of BMECs, such as claudin-1, claudin-4, occluding, and zonula occludins-1 (ZO-1), were significantly down-regulated by E. coli compared with other groups (Figure 3). Meanwhile, the expression of the four proteins was significantly up-regulated in the LCZ $+E$. coli group relative to the $E$. coli group. The expression of ZO-1 in the LCZ group was significantly higher than the expression of ZO-1 observed in the Control group; no significant differences were detected in the expression levels of the other three proteins compared with the Control group.

Effect of LCZ on the transcriptional levels of inflammatory cytokines of BMECs

The effect of LCZ on the transcriptional levels of inflammatory cytokines of BMECs is shown in Figure 4. The expression of TNF- $\alpha$, IL-6, and IL- $\beta$ was significantly up-regulated in the E. coli group relative to other groups. Although the expression levels of three inflammatory cytokines in the LCZ $+E$. coli group were significantly higher than those observed in the Control and LCZ groups, the expression levels of these cytokines were all significantly lower in these three groups relative to the $E$. coli group.

\section{Effect of $L C Z$ on $\mathbf{E}$. coli-induced impairment of mammary tissue in mice}

The mammary tissues in the Control and LCZ groups were smooth and white without any redness or swelling (Figure 5A). Redness and bleeding were visible in the E. coli group; however, in the LCZ + E. coli group, the redness and hemorrhage of the mammary tissue of mice were visibly relieved. A significant reduction in the injury score was observed in the $\mathrm{LCZ}+E$. coli group compared with the E. coli group. No significant difference in the injury score was observed among the LCZ + E. coli, LCZ, and Control groups.

No visible pathological damage was observed in the mammary acinar structure of the mice in the Control and LCZ groups. However, observable pathological injury was evident in mammary acini in the E. coligroup. In addition to damage to mammary acini structure, there was a large number of neutrophils in the alveolar lumen; in contrast, in the LCZ + E. coli group, damage to the mammary acinar structure and the number of neutrophils were visibly alleviated. A significant decrease in the histological score was observed in the $\mathrm{LCZ}+E$. coli group relative to the $E$. coli group. No significant difference in the injury score was detected between the LCZ and Control groups.

Effect of LCZ on the tight junction structure of mammary tissue in mice

The tight junction structure in the E. coli group was severely damaged, and the structure under TEM was blurred (Figure 6). However, the damage of tight junction structure in the LCZ + E. coli group was visibly alleviated. In addition, the tight junction structure in the LCZ group was clearly visible under TEM, and no observable differences were noted compared with the Control group.

Effect of LCZ on the expression of tight junction proteins of mice mammary tissue 
Tight junction proteins in mice mammary tissue, such as claudin-3, occludin, and ZO-1, were clearly localized on the interstitial side, including the intercellular regions of the neighboring alveolar epithelial cells, before E. coli injection (Control group). However, in the E. coli group, these three proteins were distributed in parts of the mammary alveolar lumen. The claudin-3, occludin, and ZO-1-positive reactions in the alveolar lumen were promoted in mice in the $\mathrm{LCZ}+E$. coli group. In the LCZ group, these three proteins were all clearly localized on the interstitial side, and no visible differences were noted compared with the Control group (Figure 7A, 7B, and 7C).

As shown in Figure 7D, the expression levels of tight junction proteins, such as claudin-3, occludin, and ZO-1, in the mammary tissues of mice were significantly down-regulated in the E. coli group compared with the other groups. The expression levels of the three proteins were significantly up-regulated in the LCZ + $E$. coli group relative to the E. coli group. No significant differences in the expression levels of the three proteins were noted between the LCZ and Control groups.

Effect of LCZ on the transcriptional levels of inflammatory cytokines of mice mammary tissue

The effect of LCZ on the expression levels of inflammatory cytokines of mice mammary tissue is shown in Figure 8. The expression levels of TNF- $\alpha$, IL-6, and IL-1 $\beta$ in the $E$. coli group were significantly up-regulated compared with the LCZ $+E$. coli group. Significant up-regulation was also observed in the expression levels of IL-1 $\beta$ in the LCZ group compared with the Control group; however, no significant differences were observed in the expression levels of IL-6 and TNF- $\alpha$.

\section{DISCUSSION}

LCZ has a promising prophylactic effect against several inflammatory diseases (Zhang et al., 2017; Wang et al., 2013; Wang et al., 2016), and recent research has shown that the oral administration of LCZ can have a prophylactic effect against $E$. coli mastitis in mice (Ma et al., 2018). Here, we performed both in vitro and in vivo tests to characterize the prophylactic effect of LCZ on E. coli -stimulated BMECs and mice mammary injury as well as elucidate its underlying mechanism. Our study showed that LCZ pretreatment could significantly ameliorate the injury of BMECs induced by E. coli, which is consistent with the findings of previous studies (Dinić et al., 2017; Wang et al., 2018; Chen et al., 2017). We also explored the prophylactic effect of LCZ on E. coli- induced mastitis in mice by intramammary injection. We found that pre-injection of LCZ could reduce the number of neutrophils and relieve damage to mammary tissue, which was also consistent with the results of our in vitro test. Recent studies have shown that intramammary injection of Lactococcusdoes not cause inflammatory reactions. After intramammary injection of Lactococcus, immune proteins, such as the acute phase proteins haptoglobin and milk amyloid A, in mammary glands of healthy Holstein cows were significantly expressed (Crispie et al., 2008; Pyorala 2003; Eckersall et al., 2006), and no major bovine mastitis pathogen was detected (Pellegrino et al., 2017). Therefore, our findings indicate that LCZ shows high potential for preventing bovine mastitis, which might be related to its ability to promote the production of lactic acid, antibacterial peptides, and other beneficial substances (Lebeer et al., 2018).

Several mammary pathogenic bacteria, including E. coli, cause mastitis. During mastitis, the blood-milk barrier becomes leaky, and molecules can cross the barrier into milk (Lehmann et al., 2013; Nguyen \& Neville, 1998). TEER reflects the integrity of the monolayer cells, the ion conductance of the pathway adjacent to monolayer epithelial cells, as well as the pore size of tight junctions (Zucco et al., 2006). Our study showed that LCZ pretreatment could significantly mitigate the reduction in TEER caused by E. coli . This result corroborated the findings of several previous studies showing that Lactobacillus could significantly promote the TEER of Caco-2 (Horibe et al., 1997) and NCM460 cells (Qiu et al., 2017; Liu et al., 2010). Accordingly, we propose that LCZ has a positive effect on enhancing the densification of monolayer BMECs. The same conclusion was obtained based on TEM observations of the desmosome structure between cells.

In exploring the underlying mechanism of the prophylactic effect of LCZ against E. coli- induced cell and blood-milk barrier damage, we found that LCZ pretreatment could significantly reduce the adhesion rate of $E$. coli to BMECs. These results are consistent with recent studies showing that Lactobacillus could significantly inhibit the adhesion of E. coli toin vitro Caco-2 (Behbahani et al., 2019) and HT-29 cells 
(Dhanani \& Bagchi, 2013). Therefore, the prophylactic effect of LCZ on BMECs could thus be mediated by inhibiting the adhesion of E. coli .

The blood-milk barrier is formed by mammary epithelial cells through special connecting structures, such as tight junctions. Tight junction proteins, such as claudin, occludin, and ZO-1, are known to maintain the structure and functional integrity of tight junctions (Guo et al., 2019; Shen et al., 2006). In this study, we found that LCZ pretreatment could significantly up-regulate the expression levels of the tight junction proteins of BMECs, which is consistent with the results of the TEER. These results are consistent with those of Karczewski et al. (2010) and Wang et al. (2018), who found that Lactobacillus plantarum could effectively promote the expression of occludin and ZO-1 in human intestinal cells as well as claudin-1, occludin, and ZO1 in IPEC-J2 cells. Moreover, Johnson et al. (2008) found that Lactobacillus rhamnosus could effectively promote the protein expression of claudin-1 and ZO-1 in T84 epithelial cells. The results of the western blot, immunofluorescence, and TEM in vivo test also showed that pre-injection of LCZ could enhance the tight junction structure and up-regulate the expression of tight junction proteins of mammary alveolar lumen in mice. This result is also consistent with our in vitro test as well as previous studies, including Patel et al. (2012), Mennigen et al. (2009), and Karczewski et al. (2010), showing that Lactobacillus can significantly promote the tightness of the intestinal epithelial barrier both in mice and humans by increasing the expression levels of tight junction proteins. Consequently, our study indicates that LCZ could promote the integrity of the epithelial barrier by up-regulating the expression of tight junction proteins in BMECs; meanwhile, intramammary injection of LCZ could effectively prevent E. coli- induced mastitis of mice from significantly down-regulating the expression of tight junction proteins in the blood-milk barrier, thereby alleviating damage to mammary tissue.

Several studies have indicated that pro-inflammatory cytokines act as important regulators in the disruption of tight junctions induced by inflammation (Schulzke et al., 2009; Zhang et al., 2018). The integrity of the blood-milk barrier can help mammary epithelial cells resist infection by external bacteria, thereby relieving the inflammatory reaction. Inflammatory cytokines, such as TNF- $\alpha$ and IL-1 $\beta$, disrupt tight junctions, and IL-6 is related to host defense against inflammatory disease (Cheng et al., 2018; Rochfort et al., 2014). Our study showed that LCZ pretreatment can significantly down-regulate the expression of TNF- $\alpha$, IL-6, and IL-1 $\beta$ induced by $E$. coli . This result is consistent with Wu et al. (2016), who found that Lactobacilluscan effectively reduce the significant up-regulation of TNF- $\alpha$, IL-1 $\beta$, IL-18, and IL- 8 mRNA expression caused by E. coli . Furthermore, previous studies have shown that the inflammatory response can cause a decrease in the expression of acinar tight junction proteins (Oguro et al., 2011; Hartwig et al., 2003) and thereby affect barrier function. Our in vitro test showed that LCZ could significantly inhibit the expression of inflammatory cytokines; consequently, we also measured the expression of inflammatory cytokines in mammary tissue in mice. We found that pre-injection of LCZ could significantly reduce the expression levels of inflammatory cytokines. Cytokines are known to play an important role in regulating the inflammatory response of the mammary gland and have important physiological and pathological effects on the blood-milk barrier. They can damage tight junction structure, thereby increasing the permeability of the epithelial barrier and causing pathogens in the acinus to enter the mammary tissue (Bruewer et al., 2006; Shen \& Turner, 2006). Therefore, our study indicates that the prophylactic effect of LCZ was achieved by inhibiting the expression of inflammatory cytokines in both BMECs and mice mammary tissue.

In conclusion, our study demonstrates that LCZ has a prophylactic effect on E. coli- induced BMEC damage and mastitis of mice by ameliorating the disruption in the blood-milk barrier disruption and suppressing inflammatory responses. The mechanism involved included the promotion of the expression of tight junction proteins by LCZ and the inhibition of the expression of important inflammatory cytokines in both BMECs and mice mammary tissue. This study provides new insights into the protective effect of LCZ, which may serve as a effective prophylactic agent to preserve the blood-milk barrier function during mastitis.

\section{Author contributions}

Y.-H. Z. performed experiments and analyzed the data. S.-L. L. supervised and validated the experiments. Y.-H. Z. interpreted the data and wrote the paper. The authors thank G. L., W. W., Y-J. W., Z-J. C., and 
H-J. Y. for their support during the study.

\section{Declaration of transparency and scientific rigor}

This Declaration acknowledges that this paper adheres to the principles for transparent reporting and scientific rigor of preclinical research recommended by funding agencies, publishers, and other organizations engaged with supporting research.

\section{References}

Angelopoulou, A., Field, D., Ryan, C. A., Stanton, C., Hill, C., \& Ross, R. P. (2018). The microbiology and treatment of human mastitis.Medical Microbiology and Immunology , 207 (5), 1-12.

Arroyo, R., Martín, V. M. A., Jiménez, E. F. L., \& Rodríguez, J. M. (2010). Treatment of infectious mastitis during lactation: Antibiotics versus oral administration of lactobacilli isolated from breast milk. Clinical Infectious Diseases , 50 (12), 1551-1558.

Behbahani, B. A., Noshad, M., \& Falah, F. (2019). Inhibition of Escherichia coli adhesion to human intestinal Caco-2 cells by probiotic candidate Lactobacillus plantarum strain L15. Microbial Pathogenesis , 136 , 103677.

Bruewer, M., Samarin, S., \& Nusrat, A. (2006). Inflammatory bowel disease and the apical junctional complex. Annals of the New York Academy of Sciences, 1072 (Aug), 242-252.

Chen, Z.-Y., Hsieh, Y.-M., Huang, C.-C., \& Tsai, C.-C. (2017). Inhibitory effects of probiotic Lactobacillus on the growth of human colonic carcinoma cell line HT-29. Molecules , 22 (1), 107.

Cheng, Y., Desse, S., Martinez, A., Worthen, R. J., Jope R. S., \& Beurel, E. (2018). TNF $\alpha$ disrupts blood brain barrier integrity to maintain prolonged depressive-like behavior in mice. Brain, Behavior, and Immunity , 69, 556-567.

Crispie, F., Alonso-Gómez, M., O’Loughlin, C., Klostermann, K., Flynn, J., Arkins, S., .. Hill, C. (2008). Intramammary infusion of a live culture for treatment of bovine mastitis: Effect of live lactococci on the mammary immune response. Journal of Dairy Research ,75 (3), 374-384.

da Costa, W. K. A., Brandão, L. R., Martino M. E., Garcia E. F., Alves, A. F., de Souza, E. L., .. Magnani, M. (2019). Qualification of tropical fruit-derived Lactobacillus plantarum strains as potential probiotics acting on blood glucose and total cholesterol levels in Wistar rats. Food Research International , 8 (35), 109-117.

Dhanani, A. S., \& Bagchi, T. (2013). Lactobacillus plantarum CS24.2 prevents Escherichia coli adhesion to HT-29 cells and also down-regulates enteropathogen-induced tumor necrosis factor-alpha and interleukin-8 expression. Microbiology $\&$ Immunology ,57 (4), 309-315.

Dinić, M., Lukić, J., Djokić, J., Milenković, M., Strahinić, I., Golić, G., .. Begović, J. (2017). Lactobacillus fermentumpostbiotic-induced autophagy as potential approach for treatment of acetaminophen hepatotoxicity. Frontiers in Microbiology ,8 (594).

Eckersall, P. D., Young, F. J., Nolan, A. M., Knight, C. H., McComb, C., Waterston, M. M., .. Fitzpatrick, J. L. (2006). Acute phase proteins in bovine milk in an experimental model of Staphylococcus aureus subclinical mastitis. Journal of Dairy Science ,89 (5), 1488-1501.

Fernández, L., Cárdenas, N., Arroyo, R., Manzano, S., Jiménez, E., Martín, V., \& Rodríguez, J. M. (2016). Prevention of infectious mastitis by oral administration of Lactobacillus salivarius PS2 during late pregnancy. Clinical Infectious Diseases ,62 (5), 568-573.

Gang L, Yin, J., Han, B., Barkema, H. W., Shahid, M., De Buck, J., .. Gao, J. (2019). Adherent/invasive capacities of bovine-associated Aerococcus viridans contribute to pathogenesis of acute mastitis in a murine model. Veterinary Microbiology ,230 , 202-211. 
Garcia-Castilla, V., Komatsu R., Clua, P., Indo, Y., Takagi, M., Salva, S., .. Villena, J. (2019). Evaluation of the immunomodulatory activities of the probiotic strain Lactobacillus fermentumUCO-979C. Frontiers in Immunology , $10,1376-1389$.

Guo, W., Liu, B., Hu, G., Kan, X., Li, Y., Gong, Q., .. Liu, J. (2019). Vanillin protects the blood-milk barrier and inhibits the inflammatory response in LPS-induced mastitis in mice. Toxicology and Applied Pharmacology , 365, 9-18.

Hartwig, W., Karen, W. B., Jörg, K., Gesa, R. E., Stefan, L., Stefan, H., .. Britta, E. (2003). Localization of claudin-3 in tight junctions of the blood-brain barrier is selectively lost during experimental autoimmune encephalomyelitis and human glioblastoma multiforme. Acta Neuropathologica , 105 (6), 586-592.

Horibe, Y., Hosoya, K., Kim, K.-J., Ogiso, T., \& Lee., V. H. L. (1997). Polar solute transport across the pigmented rabbit conjunctiva: Size dependence and the influence of 8-bromo cyclic adenosine monophosphate.Pharmaceutical Research , 14 (9), 1246-1251.

Ignacio, F., Pellegrino, M., Giraudo, J., Nader-Macias, M. E., \& Cristina, B. (2015). Evaluation of beneficial lactic acid bacteria strains as a potential probiotic for the prevention of bovine mastitis.Frontiers in Immunology , 6 (4), 231-239.

Igor, S., Sharon, M. D., Zdislava, S., Vera, N. B., Eva, V., Vera, J., .. Alla, S. (2019). Colonization of germfree piglets with commensal Lactobacillus amylovorus, Lactobacillus mucosaeand probiotic E. coli nissle 1917 and their interference withSalmonella typhimurium. Microorganisms , 7 (8), 273.

Jiménez, E., Fernández, L., Maldonado, A., Martín, R., Olivares, M., Xaus, J., .. Rodríguez, J. M. (2008). Oral administration ofLactobacillus strains isolated from breast milk as an alternative for the treatment of infectious mastitis during lactation. Applied \& Environmental Microbiology, 74 (15), 4650-4655.

Johnson, H. K. C., Donato, K. A., Shen, T. G., Gordanpour, M., \& Sherman, P. M. (2008). Lactobacillus rhamnosus strain GG prevents enterohemorrhagic Escherichia coli O157: H7-induced changes in epithelial barrier function. Infection and Immunity ,76 (4), 1340-1348.

Karczewski, J., Troost, F. J., Konings, I., Dekker, J., \& Wells, J. M. (2010). Regulation of human epithelial tight junction proteins byLactobacillus plantarum in vivo and protective effects on the epithelial barrier. American Journal of Physiology-Gastrointestinal and Liver Physiology , 298 (6), G851-G859.

Kitching, M., Mathur, H., Flynn, J., Byrne, N., Dillon, P., Sayers, R., .. Ross, R. P. (2019). A live biotherapeutic for mastitis, containing Lactococcus lactis DPC3147 with comparable efficacy to antibiotic treatment. Frontiers in Microbiology , 10 , 2220-2255.

Lhermie, G., Grohn, Y. T., \& Raboisson, D. (2016). Addressing antimicrobial resistance: An overview of priority actions to prevent suboptimal antimicrobial use in food-animal production. Frontiers in Microbiology , 7, 2114-2125.

Lebeer, S., Peter, A. M., Marco, M. L., Van Pijkeren, J.-P., Motherway, M. O., Hill, C., .. Klaenhammer, T. (2018). Identification of probiotic effector molecules: Present state and future perspectives. Current Opinion in Biotechnology , 49 , 217-223.

Lehmann, M., Wellnitz, O., \& Bruckmaier, R. M. (2013). Concomitant lipopolysaccharide-induced transfer of blood-derived components including immunoglobulins into milk. Journal of Dairy Science ,96 (2), 889-896.

Li, X., Wang, H., Du, X., Yu, W., \& Ma, C. (2017). Lactobacilli inhibit cervical cancer cell migration in vitro and reduce tumor burden in vivo through upregulation of E-cadherin. Oncology Reports ,38 (3), 1561-1568.

Liu, Z.-H., Shen, T.-Y., Zhang, P., Ma, Y.-L., Moyer, M. P., \& Qin, H.-L. (2010). Protective effects of Lactobacillus plantarumagainst epithelial barrier dysfunction of human colon cell line NCM460. World Journal of Gastroenterology , 16 (45), 5759-5766. 
Ma, C., Sun, Z., Zeng, B., Huang, S., Zhao, J., Zhang, Y., ... Zhang, H. (2018). Cow-to-mouse fecal transplantations suggest intestinal microbiome as one cause of mastitis. Microbiome , 6 (1), 200-217.

Mennigen, R., Nolte, K., Rijcken, E., Utech, M., Loeffler, B., Senninger, N., .. Bruewer, M. (2009). Probiotic mixture VSL\#3 protects the epithelial barrier by maintaining tight junction protein expression and preventing apoptosis in a murine model of colitis.American Journal of Physiology-Gastrointestinal and Liver Physiology, 296 (5), G1140-G1149.

Muziasari, W. I., Pitkänen, L. K., Sørum, H., Stedtfeld, R. D., Tiedje, J. M., \& Marko, V. (2016). The resistome of farmed fish feces contributes to the enrichment of antibiotic resistance genes in sediments below baltic sea fish farms. Frontiers in Microbiology ,7 , 2137-2147.

Nguyen, D. A., \& Neville, M. C. (1998). Tight junction regulation in the mammary gland. Journal of Mammary Gland Biology and Neoplasia, 3 (3), 233.

Oguro, M., Koike, M., Ueno, T., Asaoka, D., Mori, H., Nagahara, A., .. Watanabe, S. (2011). Dissociation and dispersion of claudin-3 from the tight junction could be one of the most sensitive indicators of reflux esophagitis in a rat model of the disease. Journal of Gastroenterology , 46 (5), 629-638.

Qiu, Y., Jiang, Z., Hu, S., Wang, L., Ma, X., \& Yang, X. (2017).Lactobacillus plantarum enhanced IL-22 production in natural killer (NK) cells that protect the integrity of intestinal epithelial cell barrier damaged by enterotoxigenic Escherichia coli .International Journal of Molecular Sciences , 18 (11), 2409-2414.

Patel, R. M., Myers, L. S., Kurundkar, A. R., Maheshwari, A., Nusrat, A., \& Lin, P. W. (2012). Probiotic bacteria induce maturation of intestinal claudin 3 expression and barrier function. The American Journal of Pathology , 180 (2), 626-635.

Pellegrino, M., Berardo, N., Giraudo, J., Nader-Macías, M. E. F., \& Bogni, C. (2017). Bovine mastitis prevention: Humoral and cellular response of dairy cows inoculated with lactic acid bacteria at the dry-off period. Beneficial Microbes , 8 (4), 589-596.

Pyorala, S. (2003). Indicators of inflammation in the diagnosis of mastitis. Veterinary Research , 34 (5), $565-578$.

Rochfort, K. D., Collins, L. E., Murphy, R. P., Cummins, P. M., \& Koval, M. (2014). Downregulation of bloodbrain barrier phenotype by proinflammatory cytokines involves NADPH oxidase-dependent ROS generation: Consequences for interendothelial adherens and tight junctions. PLoS ONE , 9 (7), e101815-e101828.

Sanders, M. E., Merenstein D. J., Reid, G., Gibson, G. R., \& Rastall, R. A. (2019). Probiotics and prebiotics in intestinal health and disease: From biology to the clinic. Nature Reviews Gastroenterology 83 Hepatology , 16 (Suppl. 1), 1-13.

Sathiyabarathi, M., Jeyakumar, S., Manimaran, A., Jayaprakash, G., Heartwin, A., Pushpadass, M., ... Dhinesh, K. (2016). Infrared thermography: A potential noninvasive tool to monitor udder health status in dairy cows. Veterinary World, 9 (10), 1075-1081.

Schulzke, J. D., Ploeger, S., Amasheh, M., Fromm, A, Zeissig, S., Troeger, H . . Fromm, M. (2009). Epithelial tight junctions in intestinal inflammation. Annals of the New York Academy of Sciences , 1165, 294-300.

Shen, L., \& Turner, J. R. (2006). Role of epithelial cells in initiation and propagation of intestinal inflammation. Eliminating the static: Tight junction dynamics exposed. American Journal of Physiology Gastrointestinal and Liver Physiology, 290 (4), G577-G582.

Shen, L., Black, E. D., Witkowski, E. D., Lencer, W. I., Guerriero, V., Schneeberger, E. E. .. Turner, J. R. (2006). Myosin light chain phosphorylation regulates barrier function by remodeling tight junction structure. Journal of Cell Science, 119 (Pt 10), 2095-2106. https://doi.org/10.1242/jcs.02915

Wang, B., Li, J., Wang, S., Hao, Y., Zhao, X., \& Chen, J. (2018).Lactobacillus plantarum ameliorates tumour necrosis factor-induced bacterial translocation in Caco-2 cells by regulation of TLR4 expression. Journal of 
Medical Microbiology , 67 (7), 982-991.

Wang, J., Ji, H., Wang, S., Liu, H., Zhang, W., Zhan, D., \& Wang, Y. (2018). Probiotic Lactobacillus plantarum promotes intestinal barrier function by strengthening the epithelium and modulating gut microbiota. Frontiers in Microbiology , 9 , 1953-1967.

Wang, Y., Li, Y., Xie, J., Zhang, Y., Wang, J., Sun, X., ... Zhang, H. (2013). Protective effects of probiotic Lactobacillus caseiZhang against endotoxin- and d-galactosamine-induced liver injury in rats via antioxidative and anti-inflammatory capacities.International Immunopharmacology , 15 (1), 30-37.

Wang Y, Xie J, Li Y, Dong, S., Liu, H., Chen, J., ... Zhang, H. (2016). Probiotic Lactobacillus casei Zhang reduces pro-inflammatory cytokine production and hepatic inflammation in a rat model of acute liver failure. European Journal of Nutrition ,55 (2), 821-831.

Wu, Q., Liu, M.-C., Yang, J., Wang, J.-F., \& Zhu, Y.-H. (2016).Lactobacillus rhamnosus GR-1 ameliorates Escherichia coli-induced inflammation and cell damage via attenuation of ASC-independent NLRP3 inflammasome activation. Applied and Environmental Microbiology ,82 (4), 1173-1182.

Zhang, M., Wu, Y., Xie, L., Teng, C.-H., Wu, F.-F., Xu, K.-B., ... Chen, D.-Q. (2018). Isoliquiritigenin protects against blood brain barrier damage and inhibits the secretion of pro-inflammatory cytokines in mice after traumatic brain injury. International Immunopharmacology , 65, 64-75.

Zhang, Y., Ma, C., Zhao, J., Xu, H., Hou, Q., \& Zhang, H. (2017).Lactobacillus casei Zhang and vitamin K2 prevent intestinal tumorigenesis in mice via adiponectin-elevated different signaling pathways. Oncotarget , 8 (15), 24719-24727.

Zucco, F., Batto, A.-F., Bises, G., Chambaz, J., Chiusolo, A., Consalvo, R., ... Stammati, A. (2006). An inter-laboratory study to evaluate the effects of medium composition on the differentiation and barrier function of Caco-2 cell lines. Alternatives to Laboratory Animals (ATLA) , 33 (6), 603-618.

\section{Figure Legends}

Figure 1: Effect of LCZ on cell growth and the blood-milk barrier. (A) The effect of LCZ on cell viability was determined by the LDH method. (B) The effect of LCZ on the change of TEER was determined using a Millicell Electrical Resistance System-2. Change of TEER represents TEER of monolayer cells after treatment - TEER of monolayer cells before treatment. (C) Transmission electron microscopy (TEM) was used to detect the structure of desmosomes. Scale bars: $1 \mu \mathrm{m}$. Data shown are means \pm SEM $(\mathrm{n}=9)$. Means with different superscripts indicate significant differences $(\mathrm{P}<0.05)$, whereas means with the same superscripts indicate treatments that are not significantly different $(\mathrm{P}>0.05)$.

Figure 2: Effect of LCZ on the adhesion rate of E. coli to BMECs. The data shown are means \pm SEM (n $=9)$. Means with different superscripts indicate significant differences $(\mathrm{P}<0.05)$, whereas means with the same superscripts indicate treatments that are not significantly different $(\mathrm{P}>0.05)$.

Figure 3: Effect of LCZ on the expression of tight junction proteins was determined by western blotting. Quantification of tight junction proteins was determined by densitometry and was normalized to $\beta$-Actin. Data shown are means \pm SEM $(\mathrm{n}=9)$. Means with different superscripts indicate significant differences $(\mathrm{P}$ $<0.05)$, whereas means with the same superscripts indicate treatments that are not significantly different $(\mathrm{P}>0.05)$.

Figure 4: Effect of LCZ on the transcriptional levels of inflammatory cytokines of BMECs. Levels of TNF- $\alpha$, IL-1 $\beta$, and IL-6 were measured by ELISA. Data shown are means \pm SEM $(n=9)$. Means with different superscripts indicate significant differences $(\mathrm{P}<0.05)$, whereas means with the same superscripts indicate treatments that are not significantly different $(\mathrm{P}>0.05)$.

Figure 5: Effect of LCZ on E. coli -induced histopathological impairment of mammary tissue in mice. (A) Injury score of mice mammary tissue from the Control (a), E. coli (b), LCZ + E. coli(c), and LCZ (d) groups. The injury score ranged from 1-5, where 1 indicated no damage, 2 indicated slight redness, 3 
indicated redness and slight bleeding, 4 indicated redness and bleeding, and 5 indicated redness and obvious bleeding. (B) Histological score of hematoxylin-eosin stained pathological sections from the Control (a),E. coli (b), LCZ + E. coli (c), and LCZ (d) groups. Hematoxylin-eosin staining of formalin-fixed mammary gland. Histopathological sections of mammary gland. Scale bars: $100 \mu \mathrm{m}$. The histological score ranged from 1-5, where 1 indicated the absence of histological features (e.g., necrosis, neutrophils, and lymphocytes), 2 indicated minimal histological features (i.e., individual neutrophils), 3 indicated mild histological features (i.e., a small amount of neutrophils), 4 indicated moderate histological characteristics (i.e., many neutrophils and slight damage to glandular structure), and 5 indicated severe histological characteristics (i.e., a large number of neutrophils and severe damage to glandular structure). Data shown are means \pm SEM $(\mathrm{n}=$ 10). Means with different superscripts indicate significant differences $(\mathrm{P}<0.05)$, and means with the same superscripts indicate treatments that are not significantly different $(\mathrm{P}>0.05)$.

Figure 6: Effect of LCZ on thee tight junction structure of mice mammary tissue. Transmission electron microscopy (TEM) was used to characterize the structure of the tight junction. Scale bars: $50 \mu \mathrm{m}$ (A) and $10 \mu \mathrm{m}(\mathrm{B})$.

Figure 7: Effect of LCZ on the expression of tight junction proteins of mice mammary tissue. (A-C) Effect of LCZ on the localization of claudin-3, occludin, and ZO-1. Blue shows nuclei (DAPI), red shows claudin-3 and occludin, and green shows ZO-1. Scale bars: $100 \mu \mathrm{m}$. (D) Prophylactic effect of LCZ on the expression levels of the tight junction proteins claudin-3, occludin, and ZO-1 was determined by western blotting. Quantification of tight junction proteins was determined by densitometry and was normalized to $\beta$-Actin. Data shown are means \pm SEM $(\mathrm{n}=10)$. Means with different superscripts indicate significant differences $(\mathrm{P}$ $<0.05)$, whereas means with the same superscripts indicate treatments that are not significantly different $(\mathrm{P}>0.05)$.

Figure 8: Effect of LCZ on the transcriptional levels of inflammatory cytokines of mice mammary tissue. Levels of TNF- $\alpha$, IL-1 $\beta$, and IL-6 were measured by ELISA. Data shown are means \pm SEM $(n=10)$. Means with different superscripts indicate significant differences $(\mathrm{P}<0.05)$, whereas means with the same superscripts indicate treatments that are not significantly different $(\mathrm{P}>0.05)$.
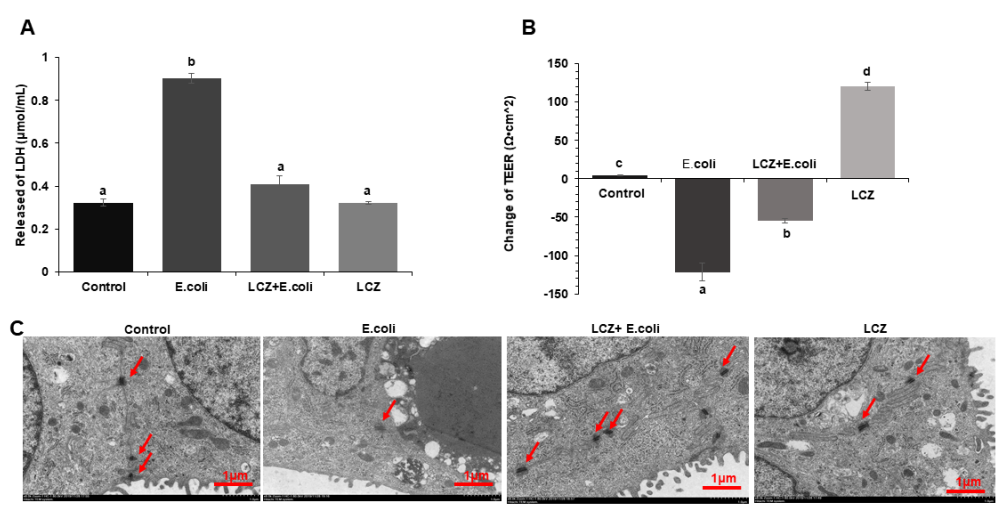


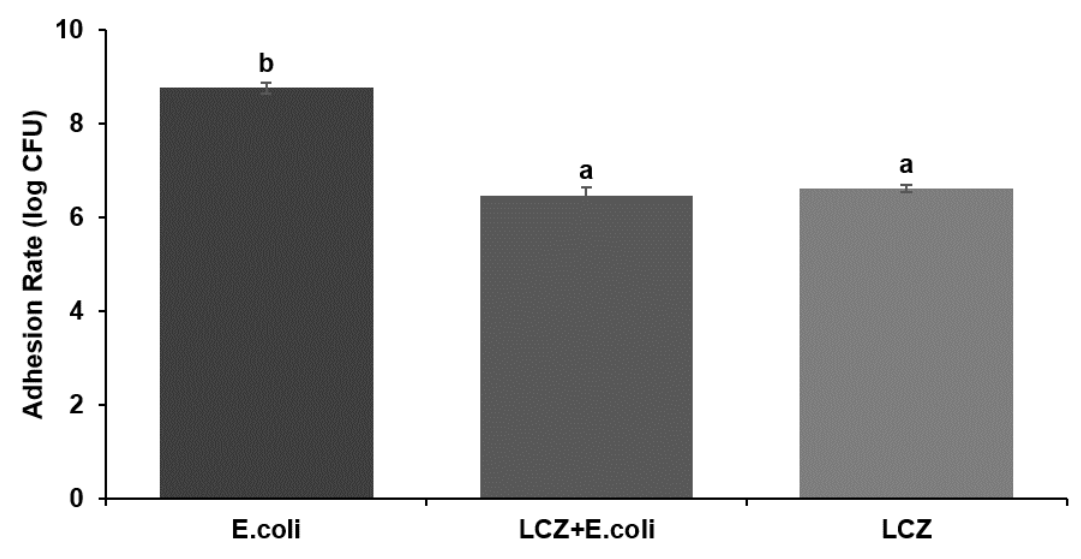

Adhesion rate of E. coli to BMECs
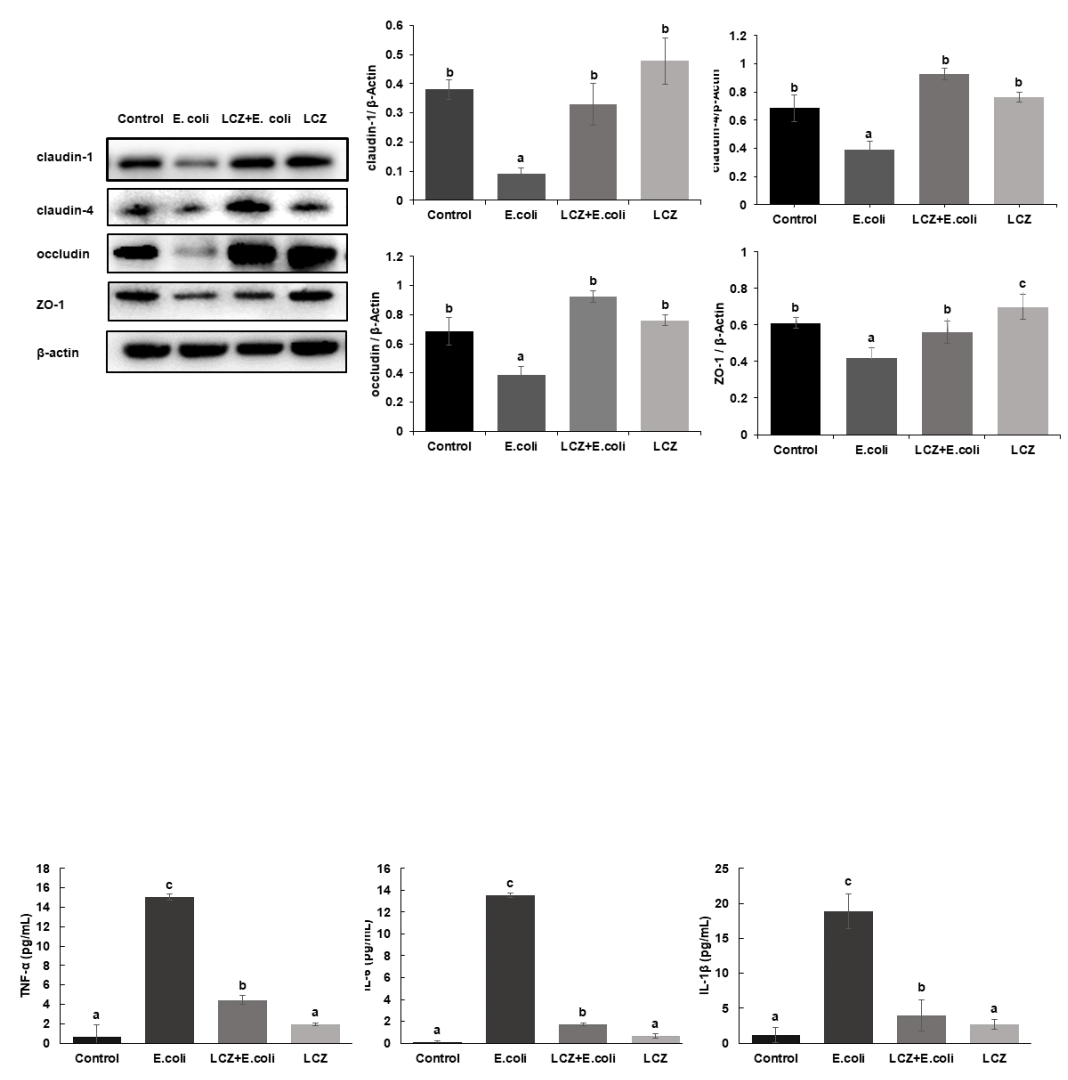

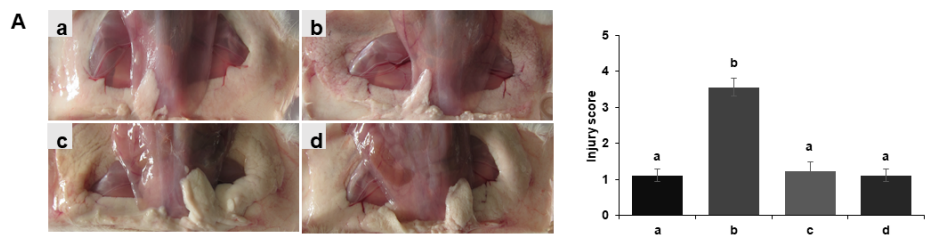

B
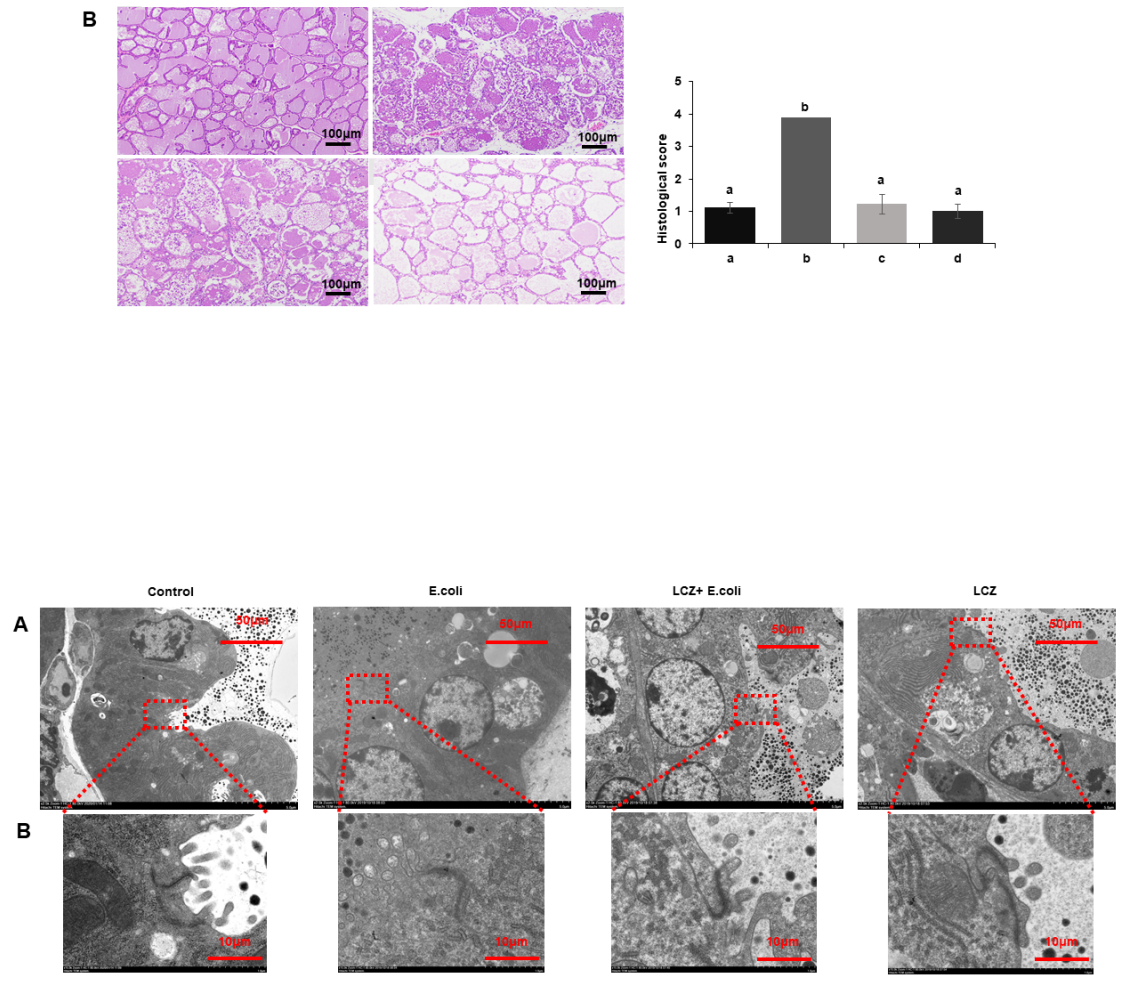

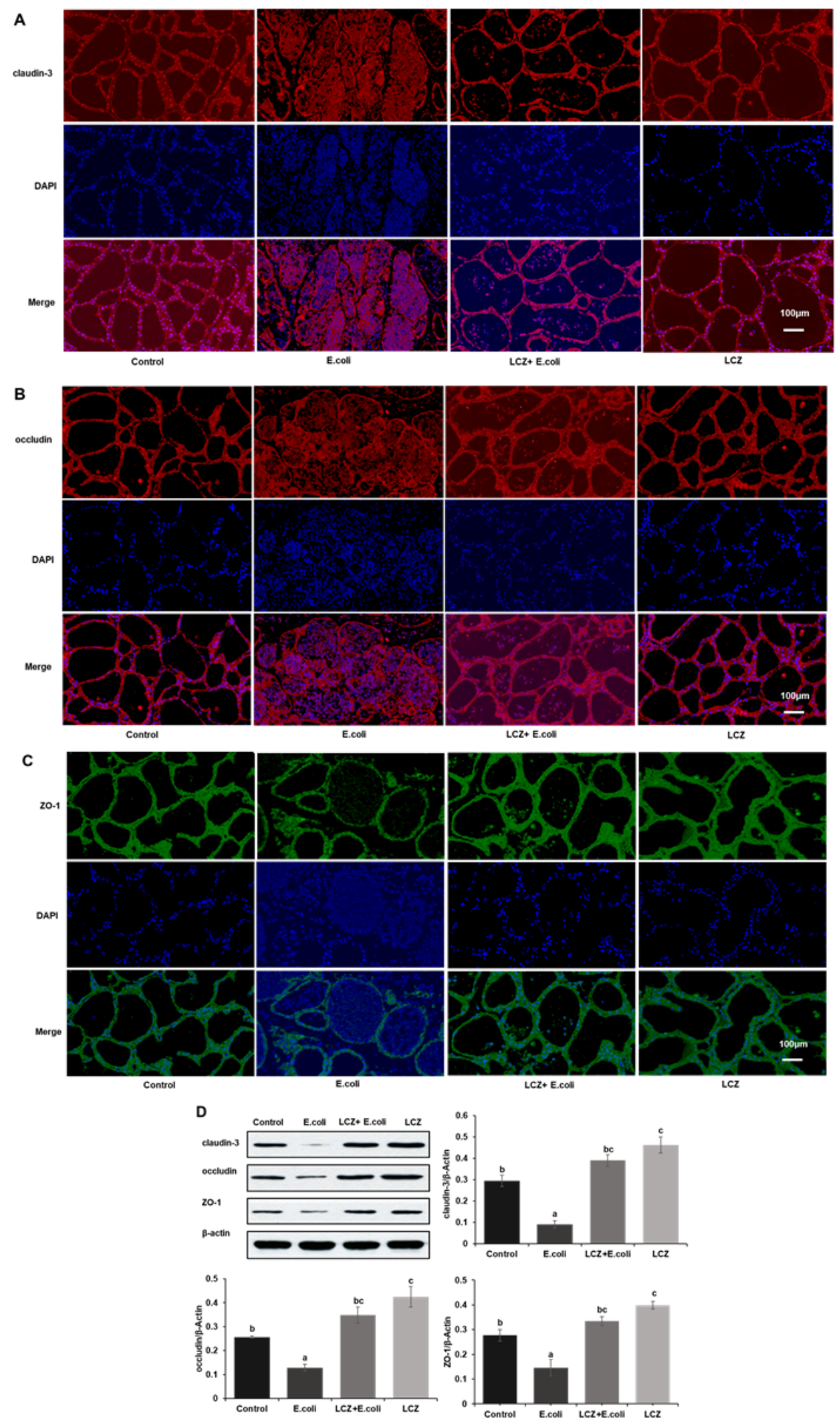


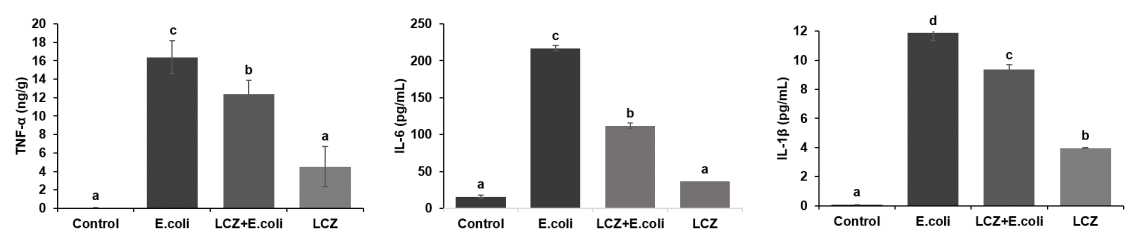

